\title{
Contribution à l'étude hydrodynamique et thermique du lac du Bourget : courants de densité et ondes internes
}

\author{
A contribution to the study of thermal and hydrodynamical behaviour \\ of lake Bourget : density currents and internal waves
}

par Pierre-Emmanuel Bournet*

CEREVE ENPC-ENGREF-UPVM, Marne-la-Vallée

- Pierre-Emmanuel Bournet a reçu une lettre de félicitations de la part du jury du prix Henri Milon.

Understanding the ecological evolution of a lake requires a good knowledge of the main physical processes which occur there. The thesis analyses two mechanisms closely associated with the hydrodynamic and thermal behaviour of lake Bourget (France) : the density currents, which are driven by the river inflows, and the internal waves, which are generated by wind events.

Two approaches have been carried out : field surveys (wind data, temperatures and currents in the lake have been collected) on the one hand, and mathematical modelling on the other hand.

Both for density currents and internal waves, the mathematical approaches have been validated for academic cases: simplified geometry, thermal stratification with two layers. At the environmental scale, numerical results are in good agreement with most of the observations. Following this study, a coupling with an ecological model will be implemented.

\section{INTRODUCTION}

Les déversements excessifs de nitrates ou phosphates liés aux développements urbains et agricoles ont fragilisé les milieux aquatiques récepteurs. L'apport de nutriments a favorisé la prolifération incontrôlée d'algues et donc l'eutrophisation. Des aménagements ont le plus souvent été effectués pour détourner ou traiter les pollutions à l'amont. Mais ces modifications ne sont pas sans conséquences sur l'évolution écologique de l'hydrosystème.

Dans ce contexte, une approche et des outils fondés sur les connaissances scientifiques apparaissent nécessaires pour limiter les impacts des aménagements des hydrosystèmes de surface, pour réduire les effets négatifs des régulations, des prélèvements ou des rejets, et enfin pour restaurer les écosystèmes dégradés en identifiant les solutions techniques appropriées.

\section{II 口OBJET ET FINALITÉ DE LA RECHERCHE ENTREPRISE}

L'analyse de l'impact de rejets polluants a été réalisée sur le lac du Bourget dont il s'agissait de faire un site pilote. Un programme environnemental pluridisciplinaire a été engagé en 1994 pour analyser et quantifier les modifications du fonctionnement physique et écologique consécutives à des variations des apports au lac.

Ce programme inclut trois volets :

1) une étude micro-biologique comprenant en particulier un suivi de la matière organique et de l'évolution des populations bactériennes ;

2) une étude physico-chimique des interactions entre l'eau du lac et les particules apportées par les affluents lors des crues ;

3) une étude hydrodynamique et thermique qui constitue l'essentiel du travail présenté.

Deux processus jouant un rôle prépondérant tant sur le plan physique que sur les plans physico-chimique et biologique ont été identifiés :

- Les courants de densité associés à l'entrée de la Leysse (le principal affluent) dans le lac du Bourget. En fonction de sa densité relativement à celle du milieu récepteur, la rivière plonge ou se propage en surface. Dans le cas de la plongée, le panache forme soit un courant de fond, soit une intrusion.

- Les ondes internes ou seiches qui consistent en des oscillations, généralement périodiques, de la thermocline. Epilimnion et hypolimnion peuvent osciller en phase, on parle alors de $1^{\text {er }}$ mode vertical. Lorsqu'ils oscillent en opposition de phase, on parle de $2^{\text {nd }}$ mode vertical. 


\section{III घINTÉRÊTT SCIENTIFIQUE}

Une bonne connaissance du compartiment physique est essentielle pour décrire le fonctionnement écologique des lacs. Les paramètres physiques influencent en effet l'évolution des paramètres physico-chimiques et biologiques du milieu. La physique intervient par le biais de l'hydrodynamique et de la thermique sous diverses formes dont notamment les courants de densité et les ondes internes.

Par advection et par entraînement, le panache modifie la distribution des nutriments, des sédiments et peut ainsi affecter la production primaire. Ainsi, l'entraînement épisodique des eaux de la rivière dans la zone euphotique peut la réapprovisionner en nutriments et maintenir la production primaire. Inversement, si le panache est très turbide, il contribue à la réduction de l'activité biologique dans la couche de surface. La rivière peut également participer à la réoxygénation des couches profondes du lac. Dans certains lacs, ce mécanisme joue un rôle prépondérant sur la dynamique interne des nutriments. Les crues ont reçu une attention particulière [1] puisqu'elles contribuent pour près de la moitié des apports annuels au lac du Bourget.

Les seiches sont responsables d'un mouvement périodique et parfois important des couches d'eau qui affecte fortement les distributions de nutriments et de biomasse et donc l'état trophique du lac. Le déplacement vertical des algues les expose en effet à des variations parfois importantes de lumière et peut ainsi influer sur leur cinétique de développement. De plus, le cisaillement qui est associé aux ondes au fond ou dans la thermocline est susceptible de favoriser la remise en suspension de particules sédimentées ou le transfert de nutriments de l'hypolimnion vers l'épilimnion. Enfin, leur déferlement sur le bord peut être à l'origine de mélange et de transport horizontal de nutriments.

\section{PROGRAMME DE TRAVAIL ET MÉTHODES EMPLOYÉES}

L'appréhension de l'écosystème lacustre a été réalisée en suivant une double approche :

- une approche de terrain qui permet de mettre en évidence les principaux mécanismes qui gouvernent l'évolution hydrodynamique et thermique du lac du Bourget ;

- une approche de modélisation qui vise à formaliser les connaissances acquises sur le fonctionnement des lacs. Plusieurs modèles de complexité croissante ont été mis en œuvre. Ils diffèrent dans la précision avec laquelle ils représentent les caractéristiques physiques du lac.

\subsection{Courants de densité}

Au niveau expérimental, une base de données incluant la température de la Leysse, les températures dans le lac à 10 niveaux entre 10 et 50 mètres et le courant à une profondeur fixée a été réalisée. Les données ont été acquises grâce à un matériel d'enregistrement automatique permettant d'échantillonner des données toutes les 10 minutes sur un mois environ. Ce matériel est constitué d'une sonde thermique pour la Leysse, d'une chaîne de thermistances et d'un courantomètre à rotor pour le lac.

La position prise par la rivière dans la colonne d'eau a été analysée en comparant les températures de la Leysse - le principal affluent - à celles du lac. On a supposé dans l'analyse que seule la température contrôlait la densité, ce qui a pu être vérifié a posteriori compte tenu de la charge relativement faible du panache en matières en suspension.
Les données ont servi de base aux orientations du travail de modélisation. Le choix du type de modèle utilisé a été dicté par les processus à représenter. En première approximation, les modèles les plus simples (de type intégral) donnent accès au chemin suivi par le panache dans le lac. En revanche, pour des épisodes particuliers à caractère exceptionnel comme les crues, une approche plus fine qui décrit l'écoulement en chaque point de l'espace a été utilisée.

Deux catégories de modèles ont été mises en œuvre : - Le modèle à tube de courant [2] intègre les équations unidirectionnelles du mouvement le long de la trajectoire du panache. Ce modèle résout les équations de conservation de la masse et de la quantité de mouvement dans un repère local suivant la ligne centrale du panache. Les variables physiques sont intégrées suivant la section transversale du panache. On distingue l'étape de propagation au fond de l'étape de propagation horizontale après décollement ;

- La propagation de la rivière dans le lac a aussi été envisagée à travers une approche originale couplant un modèle $2 \mathrm{D}$ vertical à un modèle $2 \mathrm{D}$ horizontal.

Le modèle $2 D$ vertical [3] simule la plongée et la propagation de la rivière au fond. Il repose sur une représentation de type $k-\varepsilon$ incluant les effets de flottabilité. Ce type d'approche a déjà été appliqué à l'échelle environnementale dans d'autres pays. L'étape de propagation horizontale du courant de densité après décollement du fond est décrite par un modèle $2 D$ horizontal [4]. Ce modèle à coefficient de dispersion est couplé au précédent via le débit et l'épaisseur de l'intrusion. Il néglige les échanges verticaux mais inclut les effets de la rotation de la terre. Les simulations ont été effectuées avec le code de calcul PHOENICS qui résout les équations de Navier-Stokes par une méthode aux volumes finis.

\subsection{Ondes internes}

Pour étudier les ondes internes, les mesures acquises par la chaîne de thermistances située au sud du lac ont été utilisées. Une base de données incluant les principaux paramètres météorologiques (notamment la température de l'air, la vitesse et la direction des vents) a également été mise à disposition par Météo France.

Plusieurs théories linéaires ont été appliquées afin de déterminer les caractéristiques des ondes internes en termes de période, de déplacement de l'interface de densité et de vitesse dans l'épi- et l'hypolimnion. Ces théories se distinguent par la manière dont elles prennent en compte la stratification thermique et la bathymétrie du lac. Deux des modèles utilisés assimilent le lac à un système à 2 couches homogènes - modèle de Defant [5] et méthode dite TVDM [6] - tandis que le troisième - modèle à stratification continue - [7] tient compte de la distribution réelle de densité. De façon générale, lorsque l'on améliore la représentation du profil de température, on dégrade la description de la géométrie du lac.

Pour analyser les structures frontales observées, la théorie non-linéaire développée par Korteweg et de Vries a été utilisée. Cette théorie permet en partie d'expliquer les distorsions importantes de la thermocline qui ont été observées dans le lac en période de faible stratification thermique.

\section{RÉSULTATS}

Une vingtaine de campagnes de mesures représentant près de 16 mois d'acquisition sur un peu plus de deux années d'études ont été effectuées sur le lac du Bourget.

La plupart des modèles décrits ci-dessus ont été validés pour des configurations académiques [8]. Seules seront discutées ici les applications au site d'étude qui constituent l'étape finale de mise au point des modèles. 


\subsection{Courants de densité}

Les données recueillies ont permis d'établir une comparaison de la température de la Leysse avec celle de l'air et avec celle du lac à 10 et $51 \mathrm{~m}$. Elles ont révélé que la Leysse manifeste une forte propension à former un courant de surface en été, mais plonge le restant de l'année, formant une intrusion ou un courant de fond. Cependant, compte tenu des fortes variations nycthémérales de la température de la Leysse, elles-mêmes corrélées aux fluctuations de la température de l'air, la structure du courant de densité est certainement beaucoup plus complexe que prédite, et à l'échelle de la journée, la position de la rivière peut varier sensiblement suivant la verticale. La figure 1 présente un exemple de propagation du panache dans la couche $0-10 \mathrm{~m}$.

Modèle à tube de courant : plusieurs configurations proches de cas réels de stratification ont été testées. Elles ont montré que le courant conserve une direction constante durant la phase de plongée tandis qu'il est fortement dévié vers sa droite sous l'action de la force de Coriolis après décollement du fond (fig. 2). Ces résultats sont en accord avec des observations aériennes (réalisées en 1974 lors d'une crue) qui révélaient une accumulation de matières en suspension au sud du lac.

Modèle $2 D$ vertical : quelques applications de ce modèle à l'entrée de la Leysse dans le lac du Bourget ont permis de décrire le processus de formation de l'intrusion et d'évaluer le gain de température de la rivière dû aux échanges thermiques avec le lac. La profondeur de décollement et le débit du panache évalués par ce modèle sont sensiblement identiques à ceux du modèle à tube de courant. La figure 3 présente un champ de concentration calculé en période estivale.

Modèle 2D horizontal: il a permis de déterminer de façon satisfaisante la trajectoire du panache après décollement du fond et de montrer que, conformément aux observations, le courant a tendance à suivre la rive droite du lac. L'effet d'accumulation au Sud des eaux du panache et donc d'une partie des rejets de polluants de Chambéry est restitué (fig. 4).

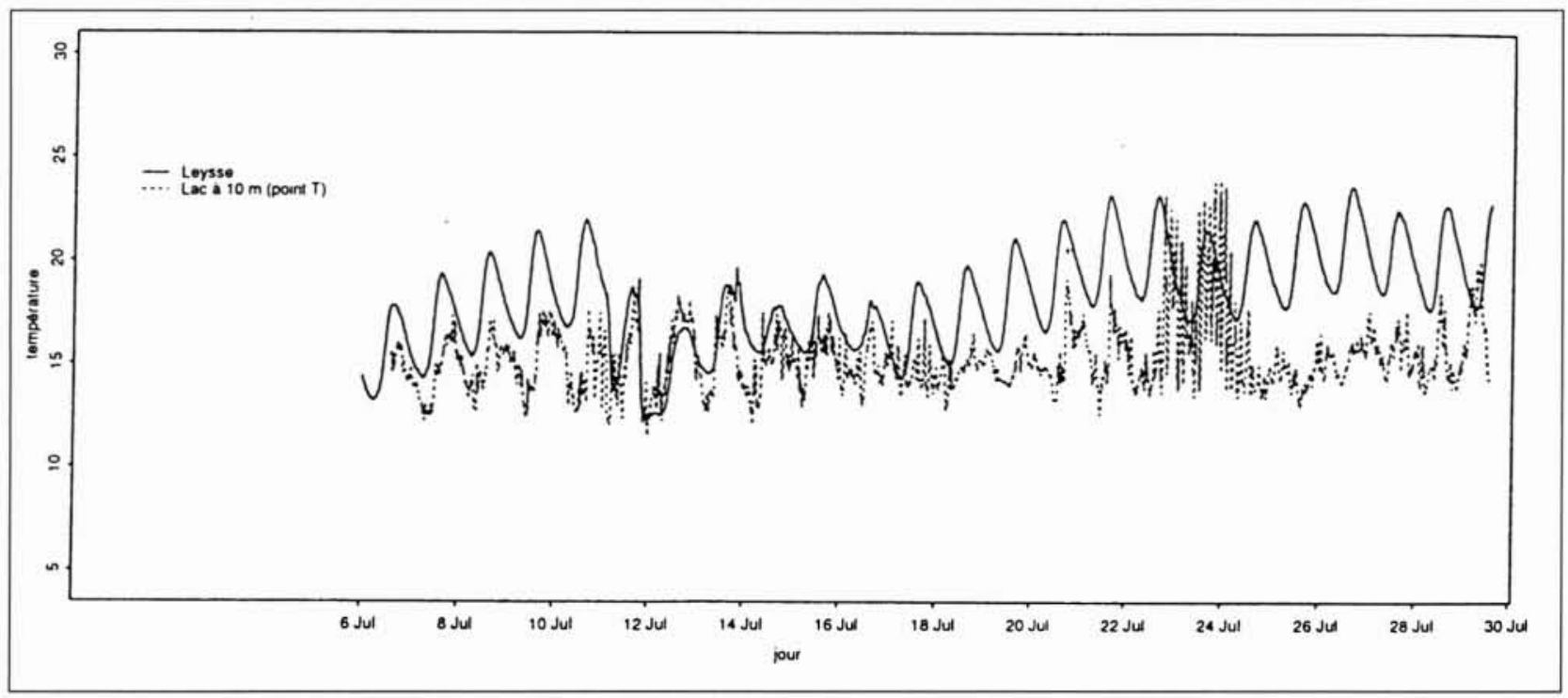

1. Comparaison des températures de la Leysse et du lac à $10 \mathrm{~m}$, juillet 1995.

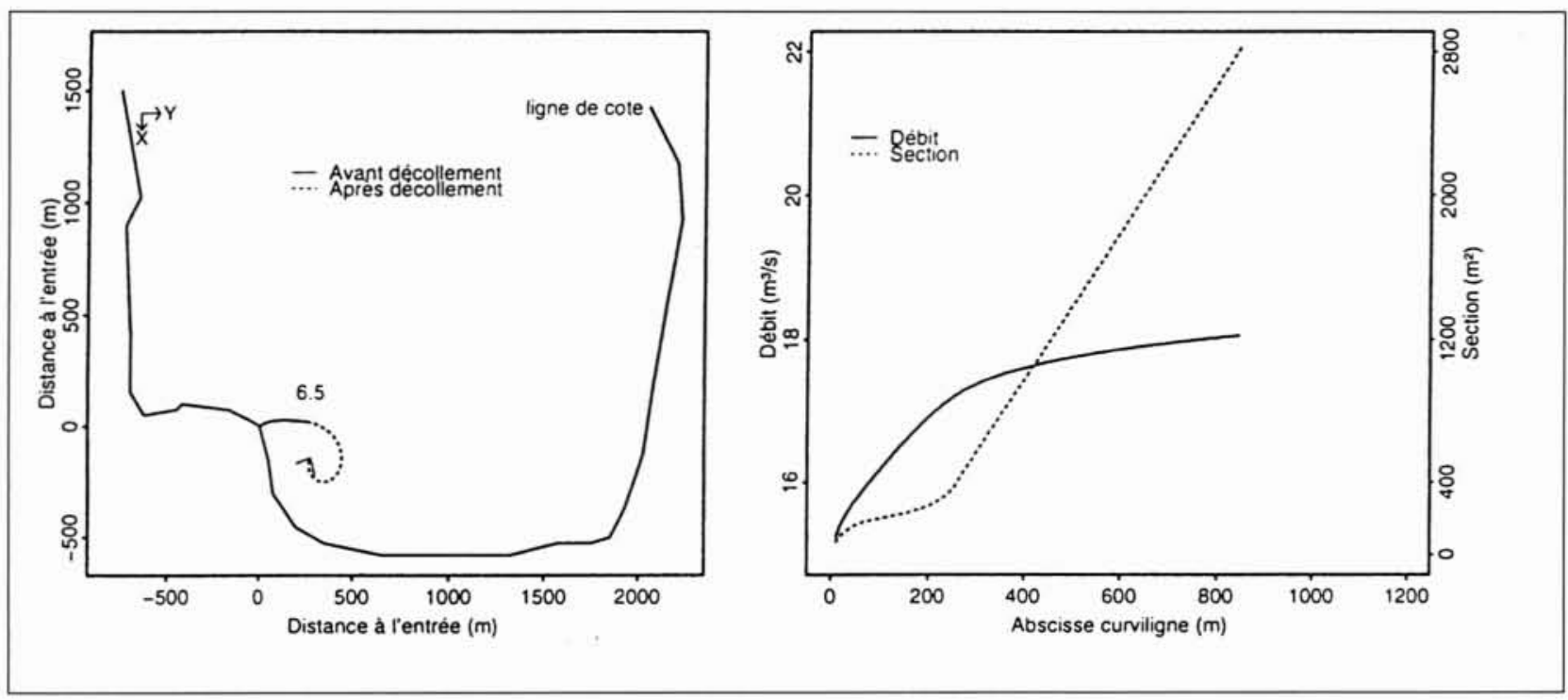

2. Trajectoire de la rivière décrite par le modèle à tube de courant ; débit et section du panache. La rivière se détache du fond à une profondeur de 6,5 m, juillet 1995 . 


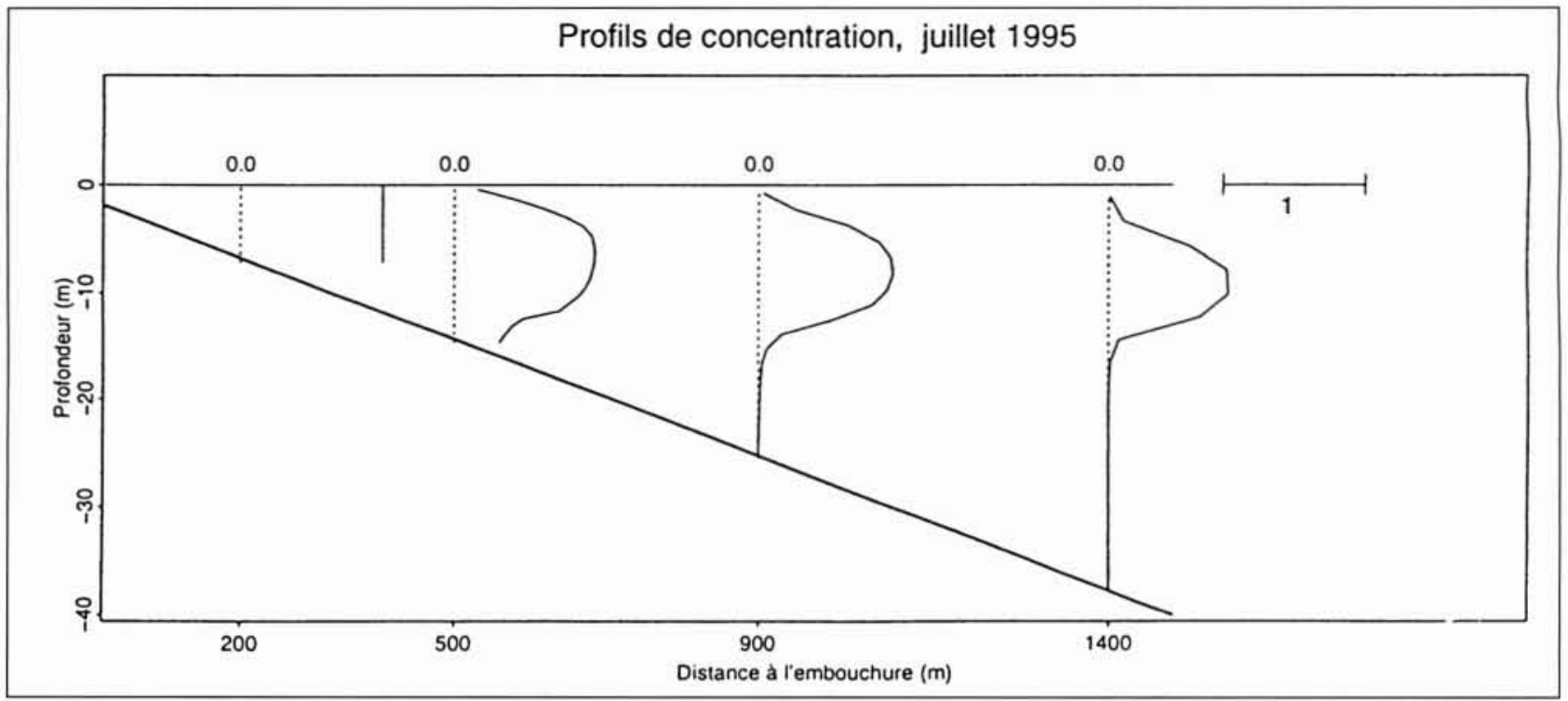

3. Profil de concentration obtenu par le modèle 2D vertical, juillet 1995.

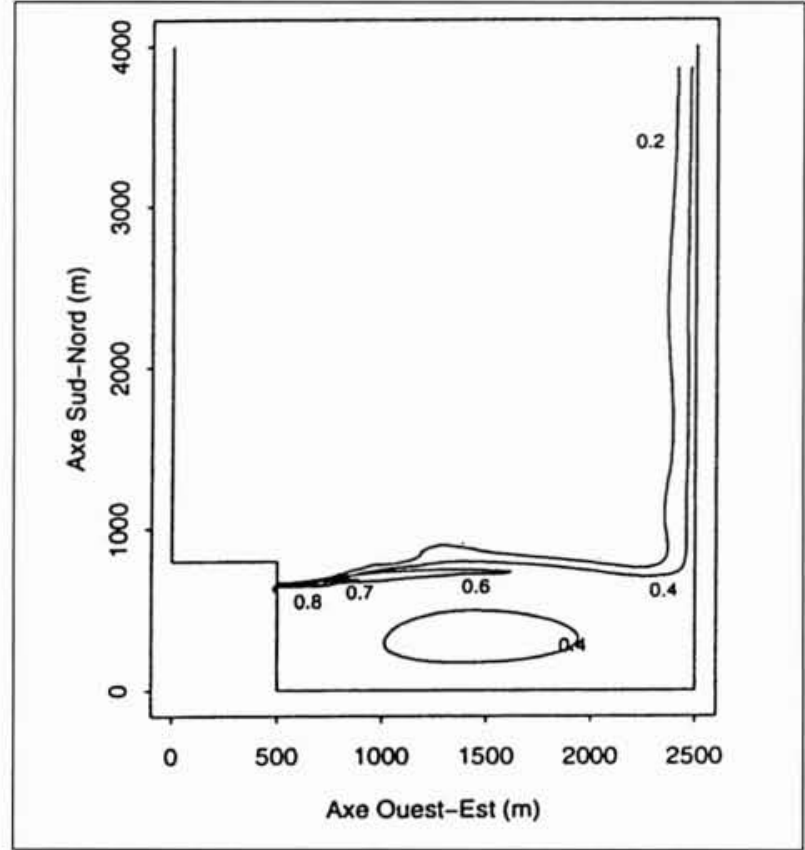

4. Champ de concentration au bout de 30 jours, modèle 2D horizontal, juillet 1995.

\subsection{Seiches internes}

Les données de températures dans le lac ont révélé l'existence de déplacements périodiques verticaux importants de la thermocline. Leur amplitude peut atteindre 40 mètres et engendrer des déplacements de masse d'eau considérables. Dans des conditions extrêmes, ces ondes peuvent déferler et contribuer au mélange de couches d'eau adjacentes.

Les modèles linéaires [8] ont mené à des résultats sensiblement identiques, en raison de la stratification marquée généralement observée et également à cause de la géométrie quasi rectangulaire du lac (fig. 5 et 6). Ils ont en particulier montré que, compte tenu du champ de vitesse calculé, un cisaillement pouvait survenir dans la thermocline et au fond et favoriser les échanges hypo-/épilimnion ou sédiment/hypolimnion.

L'analyse spectrale effectuée sur les séries temporelles de température, en complément de la modélisation mathématique, a permis de retrouver les périodes d'oscillation dominantes et d'analyser les transferts et la répartition de l'énergie dans la colonne d'eau. La période des ondes internes dans le lac du Bourget varie de $17 \mathrm{~h}$ en été à $80 \mathrm{~h}$ en automne et au printemps. Cette technique de traitement du signal constitue un moyen simple de suivre dans le plan
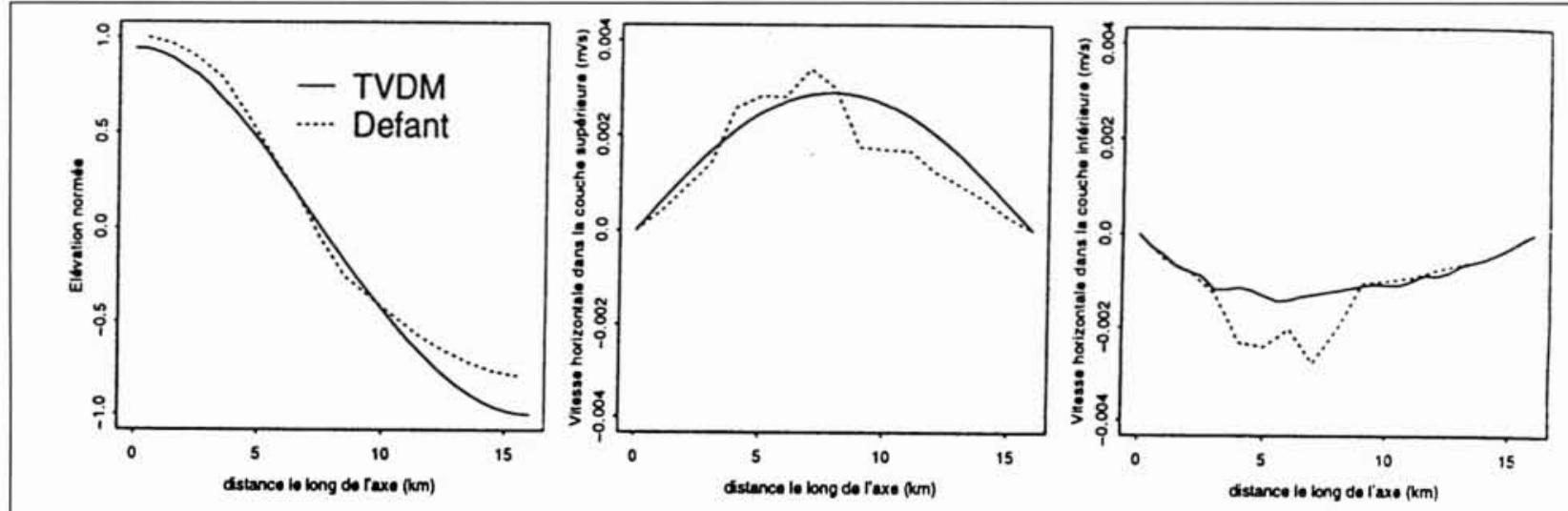

5. Résultats des modèles à 2 couches (avril 1994). 


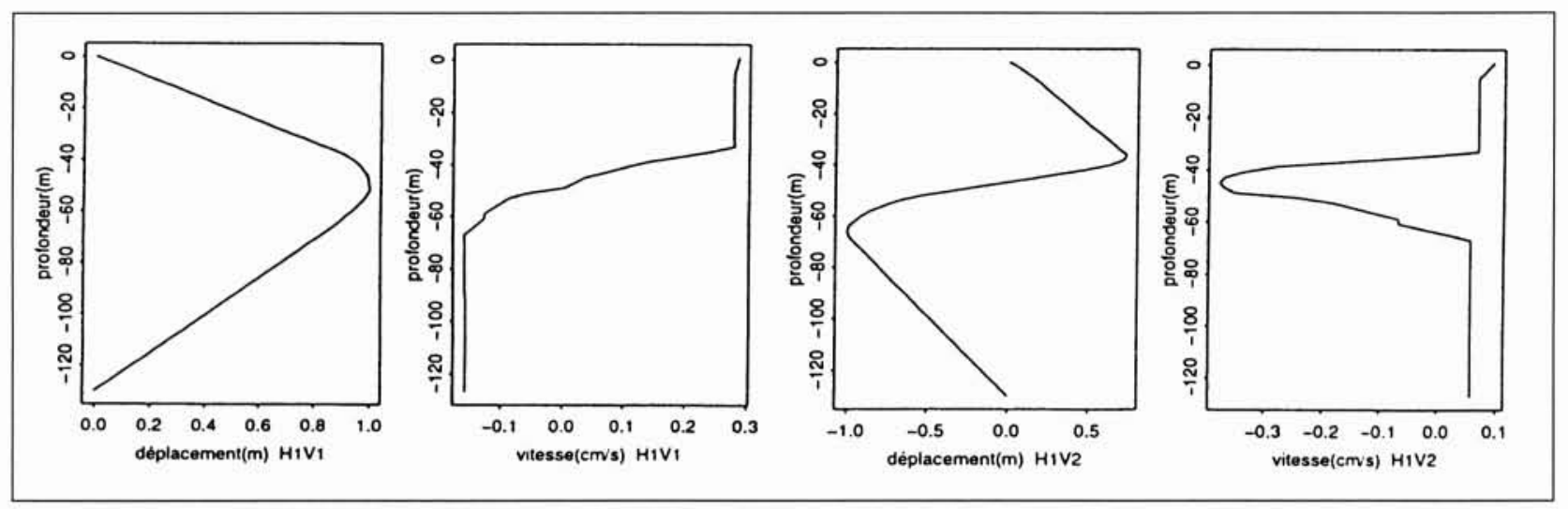

6. Résultats du modèle à stratification continue : modes 1 (H1V1) et 2 (H1V2) verticaux (avril 1994).

fréquentiel le déplacement vertical des ondes, de leur génération en avril à leur destruction en décembre. Elle confirme l'existence de distorsions importantes de la thermocline en période de faible stratification. Ces déplacements de l'interface de densité sont susceptibles de favoriser l'émergence d'instabilité de densité et donc de concourir au mélange et au transfert de nutriments à travers la thermocline.

\section{VI —CONCLUSIONS}

Le programme de recherche lancé en 1994 sur le lac du Bourget a pour objectif, à travers une approche globale pluridisciplinaire, de mieux comprendre le fonctionnement des milieux aquatiques. La thèse tente d'apporter une analyse explicative de deux phénomènes complexes : les courants de densité et les ondes internes.

Une base de données importante a été recueillie. Elle a permis de mieux cerner les processus physiques prédominants dans le lac. L'objectif de synthèse et de formalisation des connaissances via la modélisation mathématique a été atteint.

A terme, les outils développés seront intégrés dans des modèles du fonctionnement saisonnier du lac. Ils constitueront en ce sens des instruments nécessaires au gestionnaire des écosystèmes aquatiques.

\section{REMERCIEMENTS}

Ces recherches ont bénéficié du soutien du ministère de l'Agriculture.

Elles ont été menées avec le concours du Centre d'Enseigement et de Recherche sur l'Eau, la Ville et l'Environnement à Marne la Vallée, de l'Institut de Mécanique des Fluides de Toulouse, de l'Université de Savoie à Chambéry, de la Cellule technique du lac du Bourget et de la station Météo France de Voglans.

\section{RÉFÉRENCES}

[1] Vinçon-Leite B., Bournet P.E., Gayte X., FontVieille D. \& TASsin B. (1998). - «Impact of a flood event on the biogeochemical behaviour of a mesotrophic alpine lake : Lake Bourget", in Developments in Hydrobiology. Special volume Hydrobiologia. Kluwer Academic Publishers, 373/374 : 361-377.

[2] SмITH P.C. (1975), - «A stream tube model for bottom boundary currents in the ocean». Deep Sea Res., 22: 853873.

[3] Launder B.E. \& Spalding D.B. (1974). - «The numerical computation of turbulent flows». Computer Method in Applied Mechanics and Engineering, 3: 269-289.

[4] Simons T.J. (1980). - «Circulation models of lakes and inland seas». Canadian Bull. of Fisheries and Aquatic Sciences, $203: 1-146$.

[5] Lemmin U. \& Mortimer C.H. (1986). - «Test of an extension to internal seiches of Defant's procedure for the determination of surface seiche characteristics in real lakes». Limnol. Oceanogr., 31 : 1207-1231.

[6] Horn W., Mortimer C.H. \& Schwab D.J. (1986). «Wind-induced internal seiche in lake Zürich observed and modeled". Limnol. Oceanogr., 31: 1232-1254.

[7] Gill A.E. (1982). - «Atmosphere-Ocean dynamics». Academic press, San Diego, California.

[8] Bournet P.E., Dartus D., Tassin B. \& Vinçon-Leite B. (1999). - «Numerical investigation of plunging density current». J. Hydr. Eng., 125(6) : 584-594.

[9] Bournet P.E., Dartus D., Tassin B. \& Vinçon-Leite B. (1996). - «Ondes internes du lac du Bourget: analyse des observations par des modèles linéaires». Revue des sciences de l'eau, 9: 247-266. 